\title{
A Study on Arc Image Acquisition and Spectrum Inspection for Welding Process Surveillance
}

\author{
Yirong Zou*, Jiajun Liu ${ }^{2}$ and Zheming $\mathrm{Wu}^{2}$ \\ ${ }^{1}$ Tianjin Research Institute for Advanced Equipment, Tsinghua University, Tianjin, China \\ ${ }^{2}$ Tsinghua Equipment Research Institute Luoyang Base, Luoyang, China \\ ${ }^{*}$ Corresponding author
}

\begin{abstract}
This paper proposes a method for welding process surveillance, using the CCD camera and the spectrograph for the acquisition of the welding arc image and the spectral information. The spectral information inferred the temperature distribution and the metallic vapor density in the welding arc space. The physical condition of the arc space gives information about the stability of the welding process.
\end{abstract} vapor;

Keywords—welding process; spectrum; arc image; metallic

\section{INTRODUCTION}

This paper describes a study of arc image and spectrum correlation. first, through the construction of spectral acquisition and visual sensing light path, to achieve the arc space discrete point spectrum, arc space color image, arc space black and white image synchronization acquisition. The position corres pondence between spectral acquisition point and two kinds of images is calibrated by using TIG welding tungsten polar position. The Spectrum information of spectral acquisition points can be one by one corresponding to the image color component and image gray intensity of the same position in the arc space; According to the color information of the same position between the vector and the arc space. Based on the photosensitive model of color CCD pixels, based on the principal component method, the main component of the spectrum is extracted, the array of s pectral features is reduced, the mapping relation between the color information and the feature spectrum is realized, and the spatial distribution of the arc Space temperature field is calculated according to the black and white image of the arc space, and the preset boundary conditions are combined The distribution of welding current density is deduced. The studies provide a basis for the eventual establishment of a mapping relationship between arc spectral characteristics and welding metallurgical quality.

\section{SYNCHRONIZATON OF SPECTRAL AND ARC IMAGE ACQUISITION}

In order to analyze the spectral information of the welding arc space and the characteristic of the arc spatial image, the mapping relation is established. Firstly, it is necessary to realize the data acquisition of different discrete points in arc space and the sensing of the arc space image, and to ensure that the location of the spectral acquisition point can correspond to the position one by one on the arc space image. In order to ensure the consistency of welding conditions in spectral acquisition, the spectral acquisition of discrete points needs to be completed in the same welding process. Through the semi-permeable half mirror to achieve the light, black and white images and color images of the arc space for the synchronous coaxial shooting, through the convexlens, arc inverted portrait in the image plane.

Imaging adjustment process uses the image screen for positioning and adjustment. When the image is clear, the image screen is removed before the actualcollection, and the fiber head is clamped with the gripping device and the front end is positioned on the plane of the image screen. Then using the stacked manual mobile station to achieve the top and bottom of fixed-point movement, through the spectrometer to the uniform distribution of collection points for spectral information collection.

The transverse distance between the discrete points is $2 \mathrm{~mm}$, the longitudinal distance is $3 \mathrm{~mm}$, in the process of welding, manually adjust the mobile station, so that the clamp is fixed on the mobile platform to move the fiber head up and down to the designated position, and the cross-section diameter of the fiber head is less than $0.5 \mathrm{~mm}$, and the spectral collection is close to the portrait plane, the collected data can be regarded as the spectral information of the discrete points in the arc space, and are not interfered by the arc luminescence of other positions in the plane.

After the light path is built, the spectral collection of arc space discrete points, the collection of Arc color images and the collection of arc black and white images can be realized, which provides the experimental basis for the subsequent ARC spatial spectral information and image information extraction.

\section{CALCULATION OF TEMPERATURE FIELD DIST RIBUTION IN ARC SPACE}

The black and white image of welding arc can be used to calculate the temperature field of the arc space, and the calculation of the welding arc current density will provide more comprehensive information for the judgement of welding metallurgical quality. At first, the relative intensity of the arc space radiation under the characteristic spectral wavelength is obtained by the visual sensing method, according to the standard temperature method, the distribution of the temperature field in the arc space is calculated, and the conductivity distribution is obtained, based on the arc symmetry hypothesis and the 
boundary condition of the electric potential field, through the joint charge conservation equation and the Arc Space cross section current integral cons ervation equation and solved, The current density distribution of TIG arc space is obtained. The temperature field measurement method of TIG arc based on standard temperature method is mainly used to determine the relation between relative emission coefficient and temperature by using the correlation equation of inert gas ionization and the physical parameters of inert gas atoms, and then the distribution of relative emission coefficients is calculated by the gray distribution of the arc space image. After the temperature field of arc is obtained, the corres ponding physical parameters such as conductivity can be obtained in references. The optical path includes narrowband filter, $8 \mathrm{~mm}$ lens and CMOS image sensor, in which: the center wavelength of narrowband filter is $694.3 \mathrm{n} \mathrm{m}$, half bandwidth is $10 \mathrm{~nm}$, the resolution of image sensor is 480*640. The Arc temperature field calculated by the standard temperature method and the conductivity field obtained by the look-up table are shown in Figure 3.

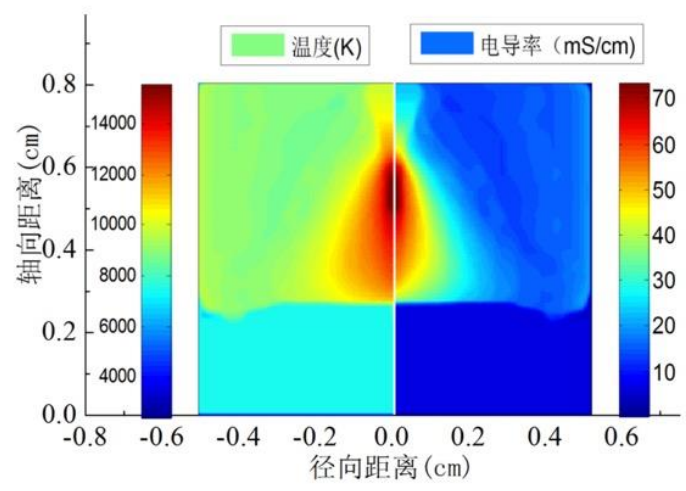

FIGURE I. ARC TEMPERATURE FIELD CALCULATED

The distribution of welding arc current density and the coupling action of heat, force and other physical quantities have great influence on the forming quality and metallurgical quality of welding.

The study of the effect of current density distribution on arc heat effects indicates that most of the thermal energy in the arc space is represented by the electron carrying and the electric current density distribution of the arc heat flux. The current density is also closely related to the ARC force during the welding process, and the arc pressure distribution is one of the key factors affecting the shape and weld forming of the molten pool. The study of arc current density can provide an important basis for studying arc welding machine and establishing numerical simulation model of weld process, and accurate measurement of arc current density distribution is of great significance to realize real-time monitoring and quality control of connection process and to realize the precise manufacture of important welding structure.

The method of measuring arc current density includes the following categories: The probe method obtains the surface current distribution by measuring the current of the probe embedded on the anode surface, and the measurement results are intuitive and easy to calculate, but the current density can be changed by the introduction of the measuring probe, and the probe is burnt seriously under the high current, which makes the application range of the method limited. The split anode method uses an insulating band to isolate the anode to two regions, by measuring the current of the total current and one region to realize the solution of current density distribution, the method is easy to implement, the data obtains the way to be reliable, but faces the arc migration problem which is caused by the temperature difference in two anode regions, The actual welding arc shape is different under the condition of non-splitting anode, the cumulative error of integral process is difficult to eliminate, which affects the accuracy of measurement; the ablation Trace analysis method calculates the current density distribution according to the area of ablation mark after the arc discharge, which is advantageous to the qualitative description of the arc shape and the estimation of the mean value of the current density of a section, However, the quantitative accuracy of the measurement results and the spatial resolution of the current density are restricted. All of the methods used in the method of invasive measurement, the welding arc current density of the experimental measurement, for the mechanism research, numerical simulation and so on to provide an important basis for research, but because of sensor devices, measuring objects and other reasons, can not be applied to the actual welding process monitoring, cannot provide real-time and accurate quality control methods for high quality welding manufacturing.

After the temperature distribution of the arc is obtained by the standard temperature method, the conductivity distribution can be calculated by look-up table, and the current density distribution is further calculated after the conductivity distribution is obtained. The solution region and boundary condition of arc space electric potential field as shown in table 1, the partial differential equation of the conductivity distribution (1), the parallel vertical (2) and (3), and then the boundary condition is solved by the finite difference method, and the distribution of electric potential field and current density is obtained. The Equation (4) is the current conservation condition of the axial section. The distribution of the current density is measured and calculated.

$$
\begin{gathered}
\frac{\partial \rho_{f}}{\partial t}+\operatorname{div}\left(j_{f}\right)=0 \\
\frac{\partial^{2} \varphi}{\partial z^{2}}+\frac{\partial^{2} \varphi}{\partial r^{2}}+\left(\frac{1}{r}+\frac{1}{\sigma} \frac{\partial \sigma}{\partial r}\right) \frac{\partial \varphi}{\partial r}+\frac{1}{\sigma} \frac{\partial \sigma}{\partial z} \frac{\partial \varphi}{\partial z}=0 \\
j_{z}=-\sigma \frac{\partial \varphi}{\partial z}, \\
j_{r}=-\sigma \frac{\partial \varphi}{\partial r} \\
\int_{0}^{R} \sigma \frac{\partial \varphi}{\partial z} r d r=\frac{I}{2 \pi}
\end{gathered}
$$

\section{ANALYSIS OF SPECTRUAL FEATURES}

According to the spectral data and the color information of the arc space, the spectral information of the low dimension is extracted by the principal component analysis method, and the 
mapping relation between the color information and the feature spectrum is studied.

Spectral information of the specified position in the arc space is obtained by spectrometer, and the distribution of the arc spatial spectra is shown in Fig. 2 and Fig. 3. Fig. 2 is the Arc spatial spectral distribution of AR arc, Fig. 3 is the Arc spatial spectral distribution of $\mathrm{He}$ arc, in which the intensity-prominent bands correspond to the characteristic spectral lines of AR and he respectively. The principal component analysis of AR arc spectral data is carried out, after calculation, the first two main components can contribute more than $99 \%$ information, that is, the first two main components can be used as a base vector to achieve all the information representation; The principal component analys is of $\mathrm{He}$ arc spectral data, after calculation, the first three principal components can contribute more than $95 \%$ of the information, that is, The first three principal components can be used as the base vectors to achieve the representation of all information. The results of the principal component analysis are shown in Fig. 4.

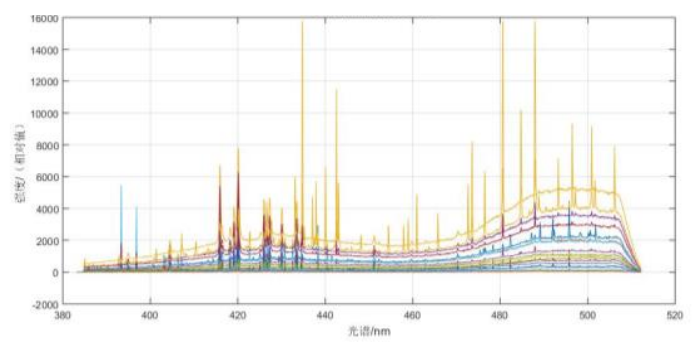

FIGURE II. SPECTRUAL INFORMATION OF AR ARC SPACE

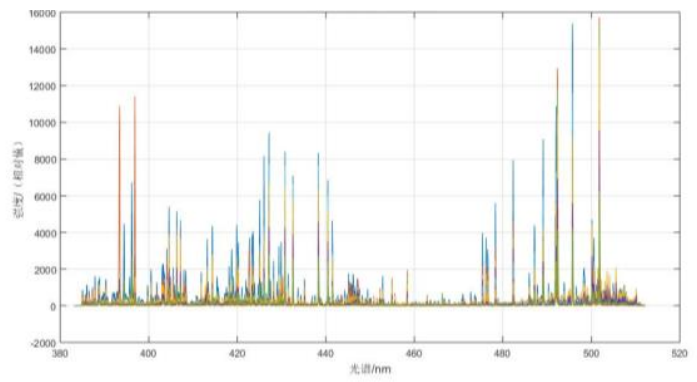

FIGURE III. SPECT URAL INFORMATION OF HE ARC SP ACE

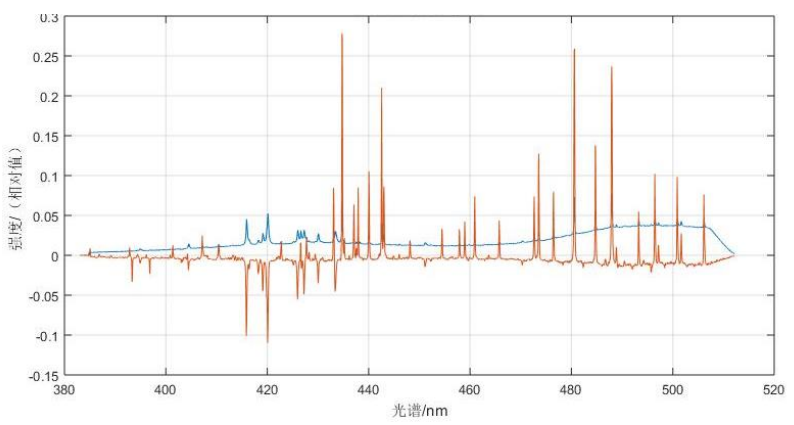

FIGURE IV. RESULTS OF PCA ANALYSIS

\section{CONCLUSION}

The current density spatial distribution of welding arc is the key factor for indicating the coupling of thermal and force and effecting the welding metallurgy and forming quality. This paper presents a method for measuring TIG arc current density spatial distribution: First, Analyzing the TIG arc image acquiring by monochromatic imaging, arc space temperature field can be calculated using the Fowler-Milne technique; Then current density spatialdis tribution can be obtained by solving the partial differential equation of electric potential according to the relationship between temperature and conductivity, the corresponding boundary conditions, the current conservation conditions. The data of current density obtained by this method have a good consistency with the literature data measured in the same experimental conditions. This method uses a non-invasive way to achieve arc current density distribution measurement and provides the technicalbas is for real-time monitoring and quality control of the welding process. The data of current density obtained by this method have a good consistency with the literature data measured in the same experimental conditions. This method uses a non-intrusive way to achieve arc current density distribution measurement and provides the technical basis for real-time monitoring and quality control of the welding process.

T ABLE I. ELECT RICAL POTENTIAL BOUNDARY CONDITIONS

\begin{tabular}{|c|c|c|c|}
\hline Area & $\begin{array}{c}\text { Boun } \\
\text { dary }\end{array}$ & $\begin{array}{c}\text { Boundary } \\
\text { condition type }\end{array}$ & $\begin{array}{c}\text { Boundary } \\
\text { condition }\end{array}$ \\
\hline \multirow{2}{*}{ C } & $\mathrm{AB}$ & Dirichlet & $\varphi=15.9$ \\
\cline { 3 - 5 } & $\mathrm{BC}$ & Neumann & $\frac{\partial \varphi}{\partial \gamma}=0$ \\
\hline & $\mathrm{CD}$ & Dirichlet & $\varphi=0$ \\
\cline { 2 - 5 } & $\mathrm{DE}$ & Neumann & $\frac{\partial \varphi}{\partial \gamma}=0$ \\
\cline { 3 - 5 } & $\mathrm{EA}$ & Neumann & $\frac{\partial \varphi}{\partial \mathrm{z}}=0$ \\
\hline
\end{tabular}

\section{ACKNOWLEDGMENT}

This study is supported by National Natural Science Funds (NO.51505252). The authors would like to thank Tianjin Research Institute for Advanced Equipment, Tsinghua University and Tsinghua Equipment Research Institute Luoyang Base for supporting the research in many aspects.

\section{REFERENCES}

[1] T sai N S, Eagar T W. Distribution of the Heat and Current Fluxes in Gas Tungsten Arcs[J],Metallurgical Transaction B. 1985. 16B.841-846.

[2] Nestor O H. Heat Intensity and Current Density Distributions at the Anode of High Current Inert Gas Arcs[J].Journal of Applied Physics, 1962,33(5):1638-1648.

[3] Shujun Chen, Fan Jiang, Zhenyang Lu,etc, Measurement and Analysis of the Welding Arc Current Density and Pressure Distribution based on Split Anode Method[C]/International Conference on Mechatronics and Aut omation. August 7 - 10, Beijing, China 1544-1549.

[4] Guangjun Zhang, Jun Xiong, Hongming Gao etc. Effect of process parameters on temperature distribution in twin-electrode T IG coupling $\operatorname{arc}[\mathrm{J}]$. Journal of Quantitative Spectroscopy \& Radiative Transfer,2012,113:1938-1945.

[5] Z. Pan, J. Polden, N. Larkin, et al. Automated Offline Programming for Robotic Welding System with High Degree of Freedoms[C]. // Y. Wu, eds. Advances in Computer, Communication, Control \& Automation, LNEE 121, 2011: 685-692. 